\title{
Correction to: Wheat wounding-responsive HD-Zip IV transcription factor GL7 is predominantly expressed in grain and activates genes encoding defensins
}

\author{
Nataliya Kovalchuk ${ }^{1} \cdot$ Wei Wu$^{1,5} \cdot$ Natalia Bazanova $^{1,6} \cdot$ Nicolas Reid $^{1} \cdot$ Rohan Singh $^{1} \cdot$ Neil Shirley $^{1} \cdot$ Omid Eini $^{2} \cdot$ \\ Alexander A. T. Johnson ${ }^{3} \cdot$ Peter Langridge $^{1} \cdot$ Maria Hrmova $^{1,4}$ D $\cdot$ Sergiy Lopato $^{1}$
}

\section{Correction to: Plant Molecular Biology https://doi.org/10.1007/s11103-019-00889-9}

Due to an unfortunate turn of events, the panels $\mathrm{O}$ to $\mathrm{S}$ are missing in Fig. 8 of the original publication. The correct
Fig. 8 and its caption is published here and should be treated as definitive.

The original article can be found online at https://doi.org/10.1007/ s11103-019-00889-9.

Maria Hrmova

maria.hrmova@adelaide.edu.au

1 School of Agriculture, Food and Wine, University of Adelaide, Glen Osmond, SA 5064, Australia

2 Department of Plant Protection, School of Agriculture, University of Zanjan, Zanjan, Iran

3 School of BioSciences, The University of Melbourne, Melbourne, VIC 3010, Australia

4 School of Life Sciences, Huaiyin Normal University, Huai' an, China

5 Present Address: Agronomy College, Sichuan Agricultural University, Ya' an 625014, China

6 Present Address: Commonwealth Scientific and Industrial Research Organisation, Glen Osmond, SA 5064, Australia 

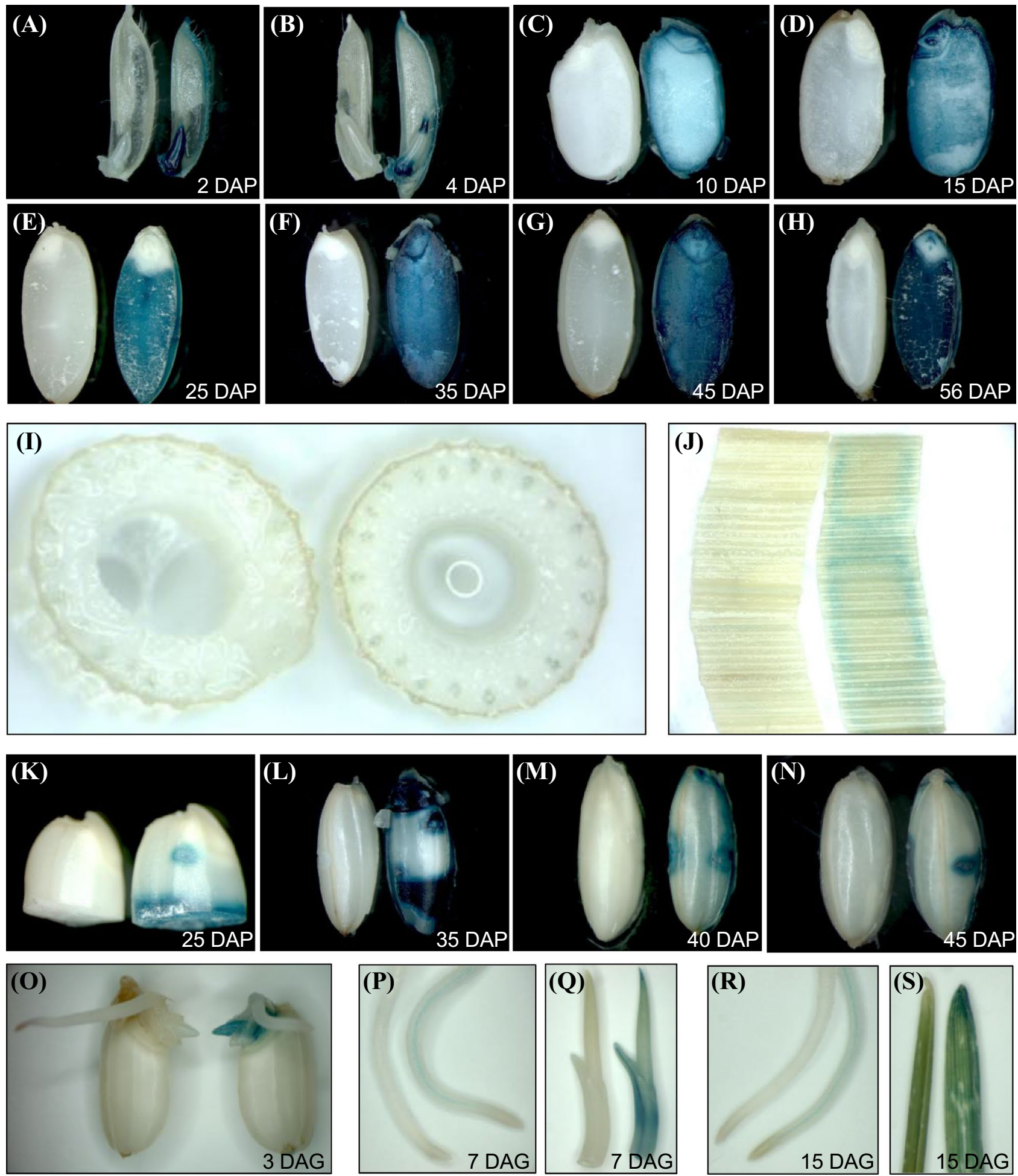

Fig. 8 GUS expression in rice directed by the $T d G L 7$ promoter. ah spatial and temporal GUS expression: a the promoter is active in ovary and upper layers of bracts at 2 DAP. $\mathbf{b}-\mathbf{h}$ Longitudinal sections of control WT and transgenic rice grains at different stages of development. i-h Induction of the $T d G L 7$ promoter by mechanical wound- ing: $\mathbf{i}, \mathbf{j}$ induction of GUS expression in vascular tissues of stem (i) and leaf (j) of rice plants. $\mathbf{k}-\mathbf{n}$ Induction of GUS in wounded areas of rice grains of different age. $\mathbf{o - s}$ GUS expression in germinating grain (o) and roots (p, r) and coleoptiles $(\mathbf{q}, \mathbf{s})$ of seedlings. DAP: days after pollination; DAG: days after germination

Publisher's Note Springer Nature remains neutral with regard to jurisdictional claims in published maps and institutional affiliations. 